\title{
Real-World Effectiveness and Safety of Lixisenatide as Add-On to Oral Antidiabetic Drugs as Part of Routine Clinical Practice in Bulgaria: LIXODAR Study
}

\author{
Nataliya Temelkova (D) - Stefka Vladeva - Aleksi Delchev • \\ Kalina Ivanova • Yoanna Gerasimova-Zheleva • Tsvetodara Kuneva • \\ Veselina Pehlivanova · Plamen Popivanov
}

Received: December 28, 2018 / Published online: March 27, 2019

(C) The Author(s) 2019

\section{ABSTRACT}

Introduction: This study aimed to demonstrate the beneficial effect of lixisenatide as add-on therapy to oral antidiabetics (OADs) in type 2 diabetes mellitus (T2DM) patients in routine clinical practice in Bulgaria.

Methods: This was a prospective, observational, multicentre study evaluating the real-life effectiveness and safety of 24-week treatment with lixisenatide in previously uncontrolled T2DM patients on combination therapy with

Enhanced Digital Features To view enhanced digital features for this article go to https://doi.org/10.6084/ m9.figshare.7844975.

Electronic supplementary material The online version of this article (https://doi.org/10.1007/s13300019-0603-9) contains supplementary material, which is available to authorized users.

N. Temelkova $(\bowtie) \cdot$ P. Popivanov

University Hospital "Alexandrovska", Sofia, Bulgaria e-mail: ntemelkova@abv.bg

S. Vladeva

University Hospital "Kaspela", Plovdiv, Bulgaria

A. Delchev

Multiprofile Hospital for Active Treatment "Prof. Dr.

Paraskev Stoyanov", Lovech, Bulgaria

K. Ivanova

Diagnostic-Consultative Centre 17, Sofia, Bulgaria metformin and sulfonylurea on highest tolerable doses.

Results: A total of 262 patients were included in the study. The mean $( \pm S D)$ age in the cohort was $56.2 \pm 9.1$ years. The mean duration of diabetes was $7.3 \pm 6.0$ years. The mean body mass index (BMI) was $39.7 \pm 4.7 \mathrm{~kg} / \mathrm{m}^{2}$. The mean glycated haemoglobin (HbA1c) at baseline was $8.8 \pm 1.1 \%$. The mean fasting plasma glucose (FPG) and postprandial plasma glucose (PPG) at baseline were $10.5 \pm 3.1 \mathrm{mmol} / \mathrm{L}$ and $12.1 \pm 3.4 \mathrm{mmol} / \mathrm{L}$ respectively. The proportion of patients achieving HbA1c $<7 \%$ at study end was $39.0 \%$ (95\% CI 32.9-45.3). The proportion of patients reaching their individual HbA1c target was $49.0 \%$ (95\% CI 42.6-55.4). The mean change in HbA1c from baseline was $-1.3 \pm 1.2 \%$. The mean change in FPG was $-2.4 \pm 3.0 \mathrm{mmol} / \mathrm{L}$ and the mean change in PPG was $-3.2 \pm 3.6 \mathrm{mmol} / \mathrm{L}$. The mean body weight change from baseline was $-7.2 \pm 5.5 \mathrm{~kg}$. The mean BMI change was

Y. Gerasimova-Zheleva

Multiprofile Hospital for Active Treatment, Silistra, Bulgaria

T. Kuneva

Diagnostic-Consultative Centre 1, Ruse, Bulgaria

V. Pehlivanova

Sanofi, Sofia, Bulgaria 
$-2.6 \pm 1.9 \mathrm{~kg} / \mathrm{m}^{2}$. The hypoglycaemia incidence was low: $6.1 \%$ for all hypoglycaemic events, $3.8 \%$ for symptomatic events and $0.4 \%$ for severe events.

Conclusions: Lixisenatide as add-on therapy to OADs in a real-life setting led to significant improvements in glycaemic control with low incidence of hypoglycaemia and beneficial weight loss. Lixisenatide was well tolerated with few patients having adverse events or discontinuing therapy. These findings are consistent with lixisenatide's safety and efficacy profile established in randomized controlled trials (RCTs).

Funding: Sanofi Bulgaria.

Keywords: GLP-1 receptor agonists; Lixisenatide; LIXODAR; Observational study; Real-life effectiveness; Routine clinical practice; Type 2 diabetes

\section{INTRODUCTION}

Type 2 diabetes mellitus (T2DM) is amongst the most common chronic diseases and is a growing worldwide epidemic [1]. In 2015, the Position Statement of the American Diabetes Association (ADA) and the European Association for the Study of Diabetes (EASD) put forth the new treatment guidance with a "patient-centred approach", with the primary goal of achieving individualized glycaemic targets whilst minimizing adverse effects, particularly weight gain and hypoglycaemia [2]. An updated consensus report on the management of hyperglycaemia in T2DM was published in 2018 by the ADA and EASD. Metformin remains the first-line therapy together with comprehensive lifestyle changes (including weight management and physical activity). If the patient cannot maintain the HbA1c target, the treatment advances to dual combination with another oral glucose-lowering agent or with a glucagon-like peptide-1 (GLP-1) receptor agonist. GLP-1 receptor agonists are recommended when there is compelling need to minimise hypoglycaemia and for patients with compelling need to minimise weight gain or promote weight loss. For patients with clinical cardiovascular disease, a GLP-1 receptor agonist with proven cardiovascular benefit is recommended. In most patients who need the greater glucose-lowering effect of an injectable medication, glucagon-like peptide 1 receptor agonists are preferred to insulin [3].

GLP-1-RAs are relatively new glucose-lowering therapeutic class, which stimulate insulin secretion and reduce glucagon secretion in a glucose-dependent manner. They may decrease gastrointestinal motility (particularly the shortacting pharmacologic compounds) and improve satiety [4]. Thus they provide significant improvement in glycated haemoglobin (HbA1c) values, with the added benefits of promoting weight loss and low risk of hypoglycaemia [5]. GLP-1 RAs are categorized as being short-acting and long-acting. Short-acting agonists predominantly affect postprandial plasma glucose (PPG) and delay gastric emptying in a sustained manner, whereas long-acting agents largely affect fasting plasma glucose (FPG) and their delay in gastric emptying appears to be subject to tachyphylaxis [6].

Lixisenatide efficacy and safety has been evaluated in the extensive clinical development GetGoal programme. In this clinical programme, lixisenatide has been shown to significantly improve glycaemic control, with low rates of hypoglycaemia and beneficial weight effects, when administered as monotherapy, as add-on therapy to oral agents, and in combination with basal insulin with or without oral antidiabetic therapy [7].

Results from the GetGoal study programme also indicate that lixisenatide has a pronounced effect on postprandial glycaemic control. Being a short-acting GLP-1 RA, lixisenatide's marked effect on postprandial blood glucose levels seems mainly to be mediated through the inhibition of gastric emptying [5].

For the evaluation of efficacy and safety, randomized controlled trials (RCT) remain the gold standard and are pivotal for regulatory bodies in the drug approval process. However, the intensive monitoring and education provided during the RCTs are not always representative of daily clinical practice. Thus, the external validity of results obtained from RCTs needs to be confirmed in a real-life setting such as non-interventional trials. Non-interventional 
observational studies with less restrictive inclusion/exclusion criteria can include broader populations of patients and offer an important tool to better assess the real-world effectiveness of a drug [8].

The objective of this study was to demonstrate the beneficial effect of lixisenatide as addon therapy to oral agents in inadequately controlled patients with $\mathrm{T} 2 \mathrm{D}$ in routine clinical practice.

\section{METHODS}

\section{Study Design}

This prospective, observational, multicentre study evaluated the real-life effectiveness and safety of lixisenatide as add-on to oral antidiabetic drugs as part of routine clinical practice in Bulgaria. Investigators were endocrinologists from inpatient and outpatient clinical centres. Participating sites (final number 40 , about $10 \%$ of all endocrinologists in Bulgaria) were equally geographically distributed across the country to ensure a good representation of the management of T2D patients on GLP-1 RA therapy. Patients were enrolled consecutively from the cohort seen by the participating physicians in their daily clinical practice. According to the non-interventional study design, all treatments and procedures were left to the physicians' discretion and did not interfere with everyday clinical practice. Lixisenatide was applied in accordance with its approved label (Summary of Product Characteristics, SmPC) and local reimbursement conditions. The data collected at each visit was part of routine patient care and no additional diagnostic procedures were applied. Collected data included demographic characteristics, diabetes history, anamnesis of microvascular and macrovascular complications, lixisenatide and oral antidiabetic (OAD) treatment, glycaemic variables, hypoglycaemic and adverse events. Microvascular complications included neuropathy, nephropathy and retinopathy. For each and every patient entering the study, investigators had to set individualized HbA1c and FPG targets based on the patient characteristics in line with ADA/EASD guidelines. Patients were asked to complete a patient diary and to document any symptomatic and/or confirmed hypoglycaemia they experienced throughout the study. Confirmed hypoglycaemia was defined as a hypoglycaemic event confirmed by a measured blood glucose level below $3.9 \mathrm{mmol} / \mathrm{L}$. Symptomatic hypoglycaemia was defined as an event with clinical symptoms with or without documented blood glucose measurement. Severe hypoglycaemia was defined as an event requiring assistance of another person to actively administer carbohydrate, glucagon or take other corrective actions. All study sites documented patient data in webbased electronic case report forms (eCRF) at inclusion and after 6 months of treatment with lixisenatide.

Patients were eligible for inclusion in the study if they fulfilled the following criteria: (a) adult patients with T2D ( $>18$ years age); (b) uncontrolled (HbA1c $>7.5 \%$ ) on combination therapy with metformin and sulfonylurea (SU) on highest tolerable doses or monotherapy with metformin or SU (in case of drug intolerance) before introduction of lixisenatide; (c) now starting lixisenatide treatment or have been treated with lixisenatide for not more than 4 weeks at the time of enrolment. Patients were excluded from the study if they had type 1 diabetes, if they were younger than 18 or older than 75 years old, if they were treated with OADs other than metformin and SU or with any insulin regimen or systemic corticosteroid, if patients were pregnant or breast-feeding, had severe gastrointestinal disease (severe gastroparesis) or creatinine clearance $<30 \mathrm{ml} / \mathrm{min}$.

The study was conducted in accordance with the principles laid down by the 18th World Medical Assembly (Helsinki, 1964) including all subsequent amendments. It was approved by the regulatory authorities and the independent ethics committee in Bulgaria. Informed consent was obtained from all individual participants included in the study.

\section{Study Endpoints}

The primary endpoint was the percentage of patients achieving HbA1c $<7 \%$ after 6 months 
of treatment. Secondary endpoints included changes in HbA1c, FPG, PPG and body weight from baseline to study end as well as frequency of confirmed and/or symptomatic hypoglycaemic events. Other secondary endpoints were the proportion of patients with at least a $0.5 \%$ and $1.0 \%$ decrease in $\mathrm{HbA} 1 \mathrm{c}$ level, proportion of patients achieving their individual $\mathrm{HbA1c}$ and FPG targets set by their treating physicians at baseline, and proportion of patients who have achieved HbA1c $<7 \%$ and have had no symptomatic hypoglycaemia and no weight gain during the 6-month treatment period.

\section{Statistical Methodology}

As a result of the observational study design, descriptive statistical methods were used including measures of central tendency and dispersion for continuous variables and numbers and percentages for categorical variables. $P$ values of these tests were interpreted in a descriptive manner. When an inferential analysis was required, independent $t$ test was used for comparison of two groups, whereas ANOVA was used in the case of more than two groups. For variables not fitting a normal distribution (based on $Q-Q$ plots), Mann-Whitney test (comparison of two groups) or Kruskal-Wallis test (comparison of more than two groups) was used. In the case of multiple comparison, Bonferroni-corrected $p$ value was reported. General linear model (GLM) regression was applied to define possible association between lixisenatide administration and FPG and PPG mean change from baseline. All tests were two-sided and with a significance level of 0.05 . The efficacy population was defined as all enrolled study patients with signed informed consent, correctly completed inclusion and exclusion criteria, and primary endpoint variables (HbA1c measurement at baseline and study end). The safety population was defined as all enrolled study patients with signed informed consent who administered lixisenatide at least once. Safetyrelated analyses (hypoglycaemic and adverse events) were performed on the safety population. All other analyses were performed on the efficacy population.
Sample size was calculated as follows: assuming that the proportion of patients achieving HbA1c $<7 \%$ would be around $20 \%$, a total number of 246 analysable patients were needed in order to obtain a 95\% confidence interval not wider than $\pm 5 \%$.

\section{RESULTS}

A total of 262 patients were included in the study between November 2015 and December 2016 and followed up by June 2017. Out of them 253 patients came back for the 6-month follow-up visit and 9 patients were lost to follow-up. Thirteen subjects (5\% of the enrolled population) had no entries related to the primary endpoint variable. Therefore 249 subjects were included in the efficacy population.

\section{Demographic and Clinical Characteristics of the Study Population}

Baseline demographic and clinical characteristics are specified in Table 1 . The mean age was $56.2 \pm 9.1$ years. The majority of patients were between 50 and 65 years old (58.6\%). Only $15.3 \%$ of the study population was at least 65 years old. The mean BMI was $39.7 \pm 4.7 \mathrm{~kg}$ / $\mathrm{m}^{2}$. The mean duration of diabetes was $7.3 \pm 6.0$ years. The mean $\mathrm{HbA1c}$ at baseline was $8.8 \pm 1.1 \%$. The majority of patients had baseline HbA1c > 8\%, with $45.8 \%$ between $8 \%$ and $9 \%$ and $27.7 \%$ greater than $9 \%$. The mean FPG at baseline was $10.5 \pm 3.1 \mathrm{mmol} / \mathrm{l}$. All 249 patients from the efficacy population had at least one PPG measurement at baseline-after breakfast, after lunch or after dinner, while $32.5 \%$ of the patients had all three measurements. The mean from all PPG measurements at baseline was $12.1 \pm 3.4 \mathrm{mmol} / \mathrm{l}$.

Clinical data related to chronic complications of T2D showed a significantly higher proportion of microvascular complications compared to macrovascular complications (Table 1). The most frequent microvascular complication was neuropathy, reported in $49.8 \%$ of the patients, while the most frequent macrovascular complication was ischemic heart disease, reported in $12.1 \%$ of the patients. 
Table 1 Baseline characteristics of the LIXODAR cohort

\begin{tabular}{|c|c|}
\hline & $\begin{array}{l}\text { Mean } \pm S D \text { or as } \\
\text { indicated }\end{array}$ \\
\hline Age, years & $56.2 \pm 9.1$ \\
\hline Males, $n(\%)$ & $119(47.8)$ \\
\hline Weight, kg & $111.2 \pm 16.0$ \\
\hline BMI, $\mathrm{kg} / \mathrm{m}^{2}$ & $39.7 \pm 4.7$ \\
\hline Diabetes duration, years & $7.3 \pm 6.0$ \\
\hline HbAlc, \% & $8.8 \pm 1.1$ \\
\hline FPG, mmol/l & $10.5 \pm 3.1$ \\
\hline PPG, mmol/l (2 h after meal) & $12.1 \pm 3.4$ \\
\hline Creatinine clearance $(\mathrm{ml} / \mathrm{min})$ & $100.7 \pm 31.8$ \\
\hline Oral antidiabetic drugs, $n(\%)$ & $249(100 \%)$ \\
\hline Metformin and SU, $n(\%)$ & $243(97.6 \%)$ \\
\hline Only metformin, $n(\%)$ & $4(1.6 \%)$ \\
\hline Only SU, $n(\%)$ & $2(0.8 \%)$ \\
\hline \multicolumn{2}{|l|}{ Microvascular complications } \\
\hline Neuropathy & $124(49.8 \%)$ \\
\hline Nephropathy & $6(2.4 \%)$ \\
\hline Retinopathy & $11(4.4 \%)$ \\
\hline \multicolumn{2}{|l|}{ Macrovascular complications } \\
\hline Myocardial infarction & $9(3.6 \%)$ \\
\hline $\begin{array}{l}\text { Stroke/transitory ischemic } \\
\text { stroke }\end{array}$ & $6(2.4 \%)$ \\
\hline Ischemic heart disease & $30(12.1 \%)$ \\
\hline Cerebrovascular disease & $9(3.6 \%)$ \\
\hline Peripheral arterial disease & $11(4.4 \%)$ \\
\hline
\end{tabular}

Overall the cohort had a high percentage of patients (45.4\%) with no complications at all.

All patients from the efficacy population received at least one OAD treatment at baseline (metformin or SU) and $97.6 \%$ of the patients received both metformin and SU (Table 1). The most frequently used OAD was metformin in $99.2 \%$ of the patients, followed by glimepiride and gliclazide MR in $41.4 \%$ and $39.4 \%$ of the patients respectively. The OAD usage at baseline and their doses can be found in Supplementary Table 1.

\section{Real-World Effectiveness Outcomes}

The proportion of patients achieving target HbA1c $<7 \%$ was $39.0 \%$ (95\% CI 32.9-45.3). The mean HbA1c at visit 2 was $7.5 \pm 1.4 \%$. The mean $\mathrm{HbA1c}$ change from baseline to the end of the 6-month follow-up period was $-1.3 \pm 1.2 \%$ (Fig. 1). A post hoc analysis showed that both proportion of patients reaching $\mathrm{HbA} 1 \mathrm{c}<7 \%$ and mean change in HbA1c depended on the baseline glycaemic control. Fewer patients tended to achieve HbA1c $<7 \%$ with the increase of baseline $\mathrm{HbA1c}$, while the mean reduction in HbA1c was greater the worse the baseline glycaemic control was (Figs. 2, 3). Glycaemic response was also stratified by diabetes

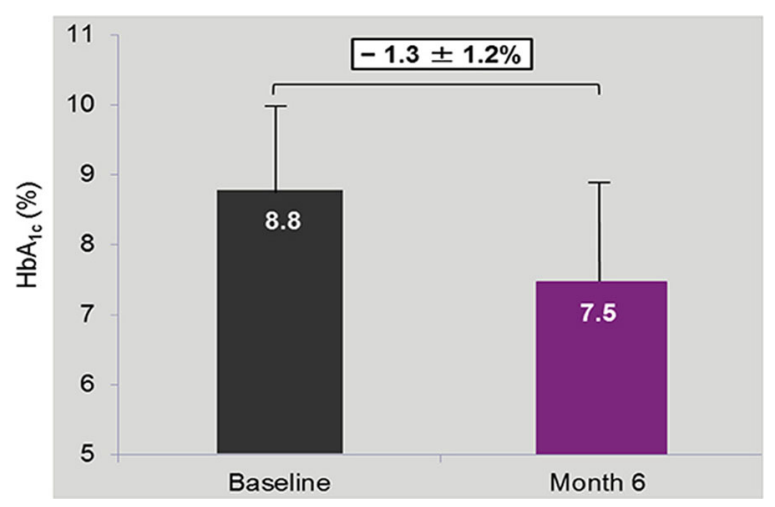

Fig. 1 Mean change in HbAlc from baseline

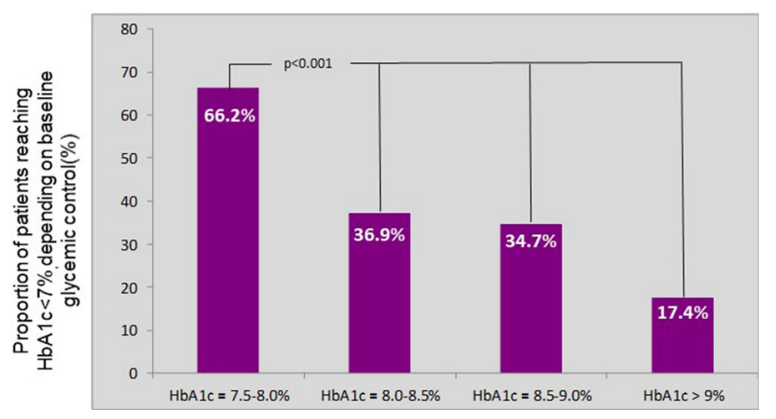

Fig. 2 Proportion of patients reaching $\mathrm{HbAlc}<7 \%$, depending on baseline glycaemic control 


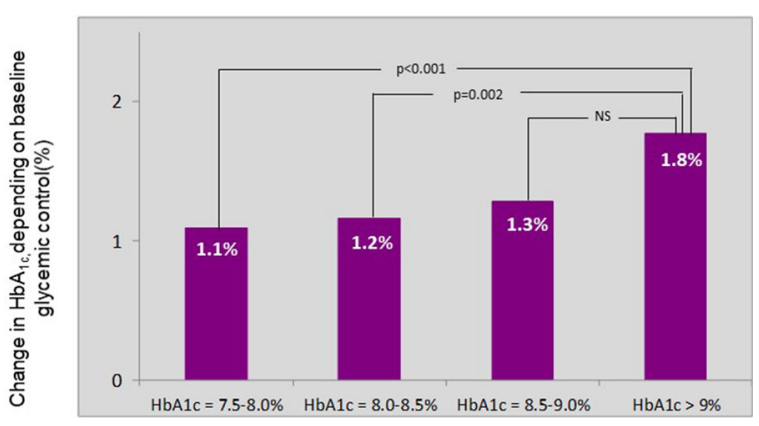

Fig. 3 Change in HbAlc, depending on baseline glycaemic control (\%)

duration. The HbA1c reduction in the patient population with shorter duration of diabetes ( $<10$ years) was $-1.4 \pm 1.2 \%$ while in the population with longer diabetes duration ( $\geq 10$ years) the reduction was $-1.2 \pm 1.2 \%$. However this difference was not statistically significant $(p=0.436)$. Similar results have been observed in regards to the proportion of patients reaching $\mathrm{HbA} 1 \mathrm{c}<7 \%$. Numerically more patients reached the target HbA1c at study end in the patient population with shorter duration of diabetes (42.3\%) compared to those in the population with longer duration of diabetes $(31.1 \%)$. However the difference was not statistically significant ( $p=0.098$ ).

A total of 245 patients had FPG measurements after 6 months of treatment. The mean FPG at study end was $8.1 \pm 3.0 \mathrm{mmol} / \mathrm{l}$. The mean FPG change from baseline was $-2.4 \pm 3.2 \mathrm{mmol} / \mathrm{l}$ (Fig. 4). Numerically the mean reduction in FPG was most pronounced

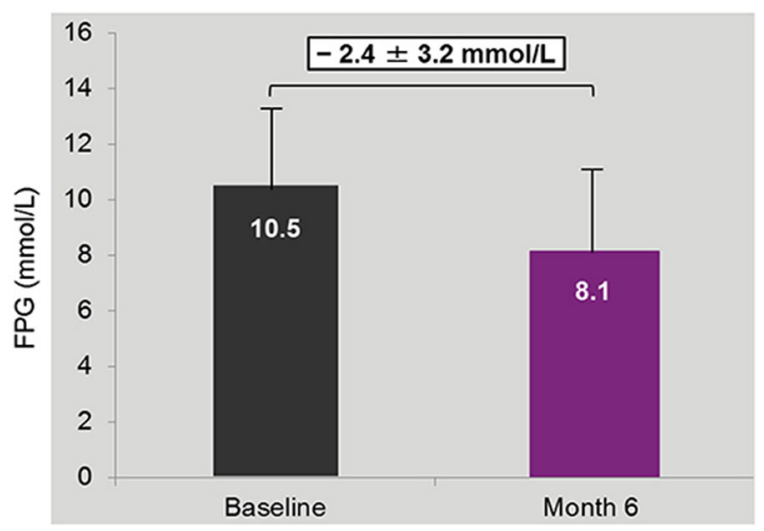

Fig. 4 Mean change in FPG from baseline when lixisenatide was administered before breakfast $(-2.7 \pm 3.5 \mathrm{mmol} / \mathrm{l})$ and before lunch $(-2.7 \pm 2.6 \mathrm{mmol} / \mathrm{l})$. When lixisenatide was administered before dinner the mean change in FPG from baseline was $-1.6 \pm 2.8 \mathrm{mmol} / \mathrm{l}$. However no statistically significant difference was observed $(p=0.124)$. Univariate analysis also showed no correlation between time of lixisenatide administration and mean change in FPG $(p=0.183)$. The mean FPG changes from baseline according to the different time of lixisenatide administration (before breakfast, lunch or dinner) are shown in Supplementary Table 2 .

A total of 242 patients had PPG measurements after 6 months of treatment. The mean PPG at study end was $8.9 \pm 3.2 \mathrm{mmol} / \mathrm{l}$ and the mean change from baseline was $-3.2 \pm 3.6 \mathrm{mmol} / \mathrm{l}$ (Fig. 5). Numerically the mean reduction in PPG was most pronounced when lixisenatide was administered before breakfast $(-3.6 \pm 3.8 \mathrm{mmol} / \mathrm{l})$ and before lunch $(-3.2 \pm 2.9 \mathrm{mmol} / \mathrm{l})$. When lixisenatide was administered before dinner the mean change in PPG from baseline was - $2.2 \pm 3.3 \mathrm{mmol} / \mathrm{l}$. Statistically significant differences were observed between the before breakfast and before dinner administration subgroups $(p=0.041)$. There was no statistically significant difference between the before lunch and before dinner subgroups $(p=0.337)$. Univariate analysis showed no correlation between time of lixisenatide administration and mean change in PPG $(p=0.586)$. The mean PPG changes from baseline according to the

Table 2 Hypoglycaemic events

\begin{tabular}{lcr}
\hline Hypoglycaemic events & $\boldsymbol{N}$ (patient) & \multicolumn{1}{c}{$\%$} \\
\hline 1 & 10 & 3.8 \\
2 & 2 & 0.8 \\
3 & 2 & 0.8 \\
4 & 1 & 0.4 \\
5 & 1 & 0.4 \\
None & 246 & 93.9 \\
Total & 262 & 100.0 \\
\hline
\end{tabular}




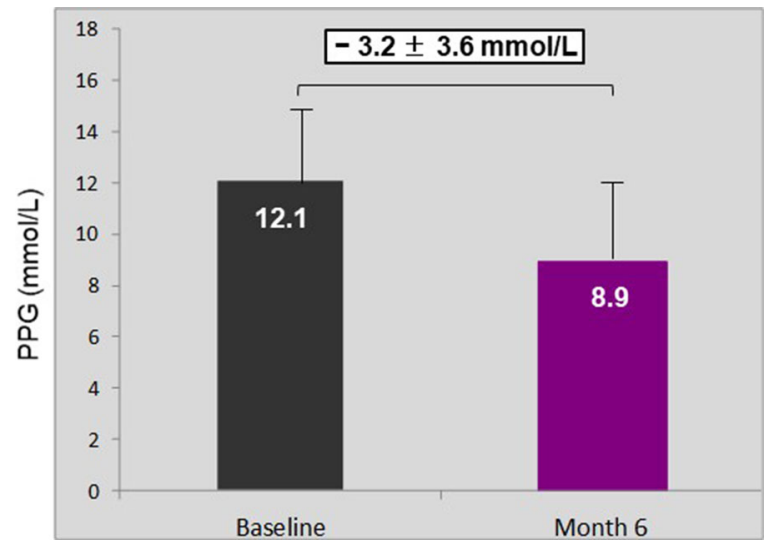

Fig. 5 Mean change in PPG from baseline

different time of lixisenatide administration (before breakfast, lunch or dinner) are shown in Supplementary Table 3 . The mean change from baseline in the different PPG measurements during the day (after breakfast, after lunch and after dinner) are shown in Supplementary Table 4.

The proportion of patients with at least a $0.5 \%$ decrease in HbA1c level was $86.7 \%$ (95\% CI 81.9-90.7). The proportion of patients with at least a $1.0 \%$ decrease in HbA1c level was 72.7\% (95\% CI 66.7-78.1).

The mean individual HbA1c target value specified by the investigators at baseline was $7.2 \pm 0.6 \%$. The mean individual FPG target value was $6.7 \pm 0.9 \mathrm{mmol} / \mathrm{l}$. Respectively $49.0 \%$ (95\% CI 42.6-55.4) of the patients reached their individualized HbA1c target and 35.1\% (95\% CI 29.1-41.4) of the patients achieved their FPG target. The majority of patients $(57 \%)$ had an individualized HbA1c target set by the investigator at baseline between $7 \%$ and $7.5 \%$. Still $27 \%$ of the patients had an individualized HbA1c target below $7 \%$, while only $10 \%$ and $6 \%$ had individualized HbA1c targets between $7.6 \%$ and $8 \%$, and more than $8 \%$, respectively. The proportion of patients reaching their individualized HbA1c target at study end according to individualized $\mathrm{HbA1c}$ target categories at baseline are shown in Fig. 6 . The majority of the patients $(85 \%)$ had an individualized FPG target set by the investigator below $7.2 \mathrm{mmol} / \mathrm{l}$ (in line with ADA/EASD guidelines). Only 14\% had individualized FPG target greater than $7.2 \mathrm{mmol} / \mathrm{l}$.

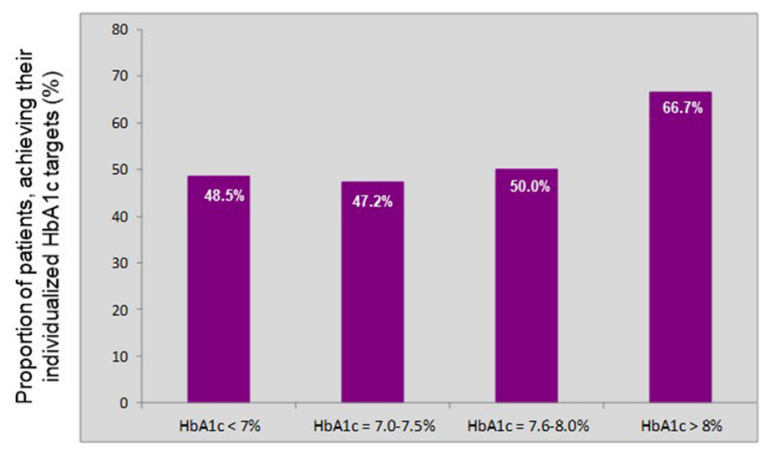

Fig. 6 Proportion of patients achieving their individualized $\mathrm{HbA} 1 \mathrm{c}$ target at study end according to individualized HbAlc target categories at baseline

The proportion of patients who have achieved HbA1c $<7 \%$ and have had no symptomatic hypoglycaemia and no weight gain for the 6-month treatment period was 36.1\% (95\% CI 30.2-42.4).

The mean body weight at study end was $104.0 \pm 15.6 \mathrm{~kg}$. The mean body weight change from baseline was $-7.2 \pm 5.5 \mathrm{~kg}$. The mean BMI at study end was $37.1 \pm 4.7 \mathrm{~kg} / \mathrm{m}^{2}$. The mean BMI change from baseline was $-2.6 \pm 1.9 \mathrm{~kg} / \mathrm{m}^{2}$. More than half of the patients managed to achieve at least 5\% weight reduction for the 6-month follow-up period, $57.8 \%$ (95\% CI 51.6-63.8). The proportion of patients with at least 3\% reduction was even higher, $83.9 \%$ (95\% CI 79.0-88.1). The proportion of patients who have achieved HbA1c < $7 \%$ with no symptomatic hypoglycaemia and at least 3\% weight reduction for the 6-month treatment period was $32.5 \%$. The proportion of patients who have achieved HbA1c $<7 \%$ with no symptomatic hypoglycaemia and at least 5\% weight reduction for the 6-month treatment period was $26.9 \%$. Weight loss and change in HbA1c were not significantly correlated (Pearson test, $p=0.173)$.

\section{Hypoglycaemia}

There were 29 hypoglycaemic events registered for 16 subjects during the study (Table 2). A total of 21 events in 10 patients were symptomatic, while 8 events in 6 patients were asymptomatic. In one patient two 
hypoglycaemic events required assistance. There was no event leading to unconsciousness/coma, or seizure, emergency department visit, or fulfilling the definition of a serious $\mathrm{AE}$. The hypoglycaemia incidence was $6.1 \%$ (16 patients) for all hypoglycaemic events, 3.82\% (10 patients) for symptomatic events and $0.38 \%$ (1 patient) for severe events. All patients who reported hypoglycaemic events throughout the 6-month follow-up period were on OAD treatment with metformin and SU.

\section{Adverse Events}

There were no AEs reported at baseline in patients who initiated lixisenatide prior to enrolment in the study. During the entire 6-month observational period, four patients from the safety population had reported six non-serious treatment emergent adverse events (two of four patients reported two events each). All AEs were of mild intensity with an outcome of recovered or resolved. One patient had allergic dermatitis (reported twice) possibly related to lixisenatide and leading to treatment discontinuation. One patient had two gastrointestinal AEs, gastritis and gastroesophagitis, possibly related to lixisenatide but the dose was not changed. For the remaining two events, the causality assessment was unrelated to lixisenatide. There was no serious adverse event reported. There were no deaths in the study.

\section{Lixisenatide Treatment}

Of the 249 patients included in the efficacy population, 248 (99.6\%) had the starting daily dose of $10 \mu \mathrm{g}$. The maintenance daily dose was $20 \mu \mathrm{g}$ for all of the patients. The most frequent daily time of administration was $1 \mathrm{~h}$ before breakfast (Table 3). At the 6-month follow-up visit 215 patients $(86.3 \%)$ continue the therapy with lixisenatide. The daily dose was $20 \mu \mathrm{g}$ for all of the patients. A total of 34 patients (13.7\%) from the efficacy population completed the study treatment period but did not continue treatment with lixisenatide after that. The most frequent reason for lixisenatide discontinuation was the patient decision in $7.2 \%$ (18) of the
Table 3 Lixisenatide daily time of administration

\begin{tabular}{lrr}
\hline Daily time of administration & \multicolumn{1}{c}{$\boldsymbol{N}$} & \% \\
\hline 1 h before breakfast & 132 & 53.0 \\
1 h before lunch & 59 & 23.7 \\
1 h before dinner & 58 & 23.3 \\
Total & 249 & 100.0 \\
\hline
\end{tabular}

patients. Other reasons, reported in 6.5\% (16) of the patients, were mainly related to reimbursement restrictions-either reduction in HbA1c for the 6-month treatment period was $\leq 0.5 \%$ and treatment continuation with lixisenatide was not approved by the health fund or patient had to start insulin treatment (at the time of the study combination of GLP- RA and insulin was not reimbursed in Bulgaria). Both patient decision and other reasons were not related to safety/tolerability issues.

\section{DISCUSSION}

Lixisenatide efficacy and safety has been comprehensively evaluated through the GetGoal clinical trial programme. Lixisenatide has been studied against placebo as monotherapy and as adjunctive therapy to both oral antidiabetics (OADs) and insulin. However real-world evidence of lixisenatide effectiveness and safety is lacking. RCT outcomes do not always mirror those seen in the real world. This observational study aimed to assess the real-life effectiveness of lixisenatide as add-on therapy to OAD in routine clinical practice in Bulgaria.

The baseline characteristics of the type 2 diabetic patients in the LIXODAR study were similar to those in the phase 3 study GetGoal-S, which evaluated the efficacy and safety of lixisenatide in type 2 patients inadequately controlled on a sulfonylurea with or without metformin [9]. However in GetGoal-S the patients were somewhat older (57.0 years), with a longer duration of diabetes (9.1 years) and less obese (BMI $30.1 \mathrm{~kg} / \mathrm{m}^{2}$ ). The patients in our study had higher baseline mean BMI due to 
reimbursement restriction criteria which limit the use of GLP-1 RA in Bulgaria only to patients with $\mathrm{BMI} \geq 35 \mathrm{~kg} / \mathrm{m}^{2}$. The mean HbA1c (8.3\%) and FPG $(9.67 \mathrm{mmol} / \mathrm{l})$ were slightly lower in the GetGoal-S study. In GetGoal-S the majority of patients $(84 \%)$ were receiving metformin in addition to their SU therapy at baseline [9].

In this observational study the beneficial effect of lixisenatide on the glycaemic control, as add-on therapy to OAD-based antidiabetic regimen in routine clinical practice, was measured by the proportion of patients achieving HbA1c $<7 \%$. At the end of study the proportion of patients achieving target HbA1c $<7 \%$ was $39.0 \%$. Similar results were observed in the GetGoal-S study where the HbA1c target of $\leq 7.0 \%$ was achieved by $36.4 \%$ of the patients [9]. Of note is that a slightly higher percentage of patients achieved HbA1c $<7 \%$ in the LIXODAR cohort even though the baseline HbA1c was higher compared to the baseline HbA1c in GetGoal-S.

In our study the mean HbA1c change from baseline was $-1.3 \%$. This reduction in HbA1c is greater compared to the GetGoal-S study where the mean change from baseline to week 24 was - $0.9 \%$. The reduction observed in the LIXODAR study was actually greater compared to all phase 3 studies evaluating lixisenatide after OAD failure [9-12]. Interestingly the mean reduction in HbA1c observed in the LIXODAR study was greater compared to some observational studies with other GLP-1 receptor agonists. A recent retrospective cohort study using a large US claims database showed that realworld T2D patients initiating a GLP-1RA experienced smaller reduction in HbA1c $(-0.52 \%)$ than reported in RCTs $(-1.30 \%)$ after 12 months of treatment [13]. The results of the US database study can be explained by the low compliance of patients treated with GLP-1RAs. Only $29 \%$ of the patients were adherent to their medications, much lower than would be expected in RCTs. On the contrary, in our study patient compliance was good. Only $7.2 \%$ of the patients discontinued lixisenatide before the 6-month follow-up visit because of their decision. Moreover in all of the patients continuing lixisenatide treatment the maintenance dose was $20 \mu \mathrm{g}$. Another retrospective cohort study evaluating the real-world effectiveness of liraglutide and lixisenatide in UK also showed smaller reductions in HbA1c in T2D patients initiating a GLP-1RA in routine clinical practice [14]. In that study the observed change in HbA1c for 12 months was - 0.93\% with liraglutide and $-0.70 \%$ with lixisenatide. This response is even lower having in mind the high mean baseline HbA1c in the cohort $(9.49 \%$ and 9.64\% for liraglutide and lixisenatide respectively). For comparison in our study the change in HbA1c in the population with baseline HbA1c $>9 \%$ was $-1.8 \%$. Unfortunately patient compliance and adherence to therapy, which might be a possible explanation for this lower response, are not reported in the UK study.

In a post hoc analysis we have stratified the glycaemic response (patients achieving target HbA1c $<7 \%$ and mean change in HbA1c) by baseline glycaemic control and diabetes duration. Both parameters evaluating the glycaemic response depended on baseline glycaemic control but did not depend on diabetes duration. Different results have been reported in the UK retrospective study which showed a poorer response to the glycaemic reduction and control targets with increased diabetes duration. This finding is consistent with previous data reported for liraglutide; however, there is not much data supporting this finding for lixisenatide. This could be explained by the fact that lixisenatide as a short-acting GLP-1 RA predominantly affects PPG through the inhibition of gastric emptying, which is independent from the insulin secretion decrease observed with the progress of T2D.

In the LIXODAR study, in addition to improved HbA1c, there was also a decrease in both FPG and PPG values at study end compared to baseline. The mean FPG change from baseline was $-2.4 \mathrm{mmol} / \mathrm{l}$. Again, the reduction in FPG, observed in our study, is greater compared to all phase 3 studies evaluating lixisenatide after OAD failure, where the mean change in FPG from baseline to week 24 was between - $0.8 \mathrm{mmol} / \mathrm{l}$ and - $1.3 \mathrm{mmol} / \mathrm{l}$ [9-12]. Improvement in all postprandial blood glucose measurements was also observed. Results showed that reduction was the largest after breakfast and lunch. These results correspond to 
the time of administration of lixisenatide since $53 \%$ of patients have administered lixisenatide prior to breakfast and another $23.7 \%$ prior to lunch in our study. However no correlations were proved between the time of lixisenatide administration and mean change in FPG and PPG. Overall the decrease in PPG was more pronounced as compared to the FPG decrease observed. Results from the GetGoal study programme also indicate that lixisenatide has a pronounced effect on postprandial glycaemic control. Compared with placebo, significant decreases (of $3.2-7.2 \mathrm{mmol} / \mathrm{L}$ ) in PPG from baseline to week 24 have been observed. The recent recommendations indicate that fasting and pre-meal glucose should be maintained at $<7.2 \mathrm{mmol} / \mathrm{L}$ and the postprandial glucose (PPG) at $<10 \mathrm{mmol} / \mathrm{L}$ with a patient-centred approach [2]. In our study all mean PPG values at study end were $<10 \mathrm{mmol} / \mathrm{L}$; the mean PPGs after breakfast, lunch and dinner were $9.0 \mathrm{mmol} / \mathrm{l}, 8.7 \mathrm{mmol} / \mathrm{l}$ and $9.0 \mathrm{mmol} / \mathrm{l}$ respectively. This means that the PPG has been reduced to target values.

The proportion of patients with at least a $1.0 \%$ decrease in HbA1c level was relatively high (72.7\%). Another recent retrospective cohort study using administrative claims data showed that adherent real-world T2D patients on liraglutide were more likely to have at least a $1.0 \%$ decrease in their HbA1c compared to the non-adherent cohort (38\% vs. $32 \%, p=0.022)$ [15]. Still the proportion of patients experiencing a $1.0 \% \mathrm{HbA} 1 \mathrm{c}$ decrease in both cohorts was much smaller compared to our study results. The results from our study are much closer to the retrospective UK cohort study where the proportion of patients with at least $1.0 \%$ decrease in $\mathrm{HbA} 1 \mathrm{c}$ was $76.0 \%$ for liraglutide and $63.6 \%$ for lixisenatide [14]. Still we need to have in mind the higher baseline HbA1c in the UK cohort which would suggest greater glycaemic response.

In the RCTs the patients are always treated to target $\mathrm{HbA} 1 \mathrm{c} \leq 7 \%$. However in real life, in line with the patient-centred approach supported by the international guidelines, not all the patients are treated to $\mathrm{HbA} 1 \mathrm{c} \leq 7 \%$. It is interesting to note that in the LIXODAR study the mean individual $\mathrm{HbA1c}$ target value specified by the investigators at baseline was $7.2 \pm 0.6 \%$ which is quite close to the universally accepted target. The mean individual target value of the FPG was $6.7 \pm 0.9 \mathrm{mmol} / \mathrm{l}$ which is even lower than the recommended FPG threshold. The results showed that $49.0 \%$ of the patients reached their individualized HbA1c target and $35.1 \%$ of the patients achieved their fasting blood glucose target.

In addition to its beneficial effects on improving glycaemic control, treatment with lixisenatide has been shown to result in reductions in body weight compared with placebo. Similarly in our study the average body weight and BMI were reduced at the end of the observational period compared to baseline. Additionally this decrease was much greater compared to the phase 3 studies evaluating lixisenatide after OAD failure [9-12]. It should be noted that the population in the LIXODAR cohort was more obese than in GetGoal studies due to reimbursement restriction criteria which limit the use of GLP-1 RA in Bulgaria only to patients with $\mathrm{BMI} \geq 35 \mathrm{~kg} / \mathrm{m}^{2}$. Therefore a significant effect on body weight has been observed in this real-world study. The proportion of patients with at least 3\% reduction was also very high. Actually it was approximately twice the proportion of patients with at least 3\% weight reduction in the UK retrospective cohort study (44.9\% for the liraglutide and $40.8 \%$ for the lixisenatide group). It is an interesting finding as the mean BMI in the UK study was also very high $\left(38 \mathrm{~kg} / \mathrm{m}^{2}\right)$. The better patient compliance and adherence to therapy in our study might be a possible explanation for this better response both in terms of HbA1c and weight reduction. The GetGoal programme showed that when administered as an add-on to OAD therapy, lixisenatide does not increase the frequency of hypoglycaemia compared with placebo and the reported rates for severe hypoglycaemia are very low [9-12]. Similarly the hypoglycaemia incidence in our study was very low. Overall safety findings were consistent with lixisenatide's established safety profile.

The present study is subject to some limitations. These include the observational nature of the study and lack of a comparator arm, which have the potential to introduce bias and 
confounding into the analysis. The population in the study was obese, which could limit extrapolating the results to other populations with lower BMI. In contrast, the strengths of the LIXODAR study include the large real-world patient population and the prospective multicentre design of the study. The prospective follow-up of frequency of hypoglycaemia and adverse events ensures better reliability of data compared to retrospective real-world studies.

\section{CONCLUSION}

The current prospective observational study provides insights into the effectiveness and safety of lixisenatide as add-on therapy to OADbased antidiabetic regimen in clinical practice in Bulgaria.

Treatment with lixisenatide when added to uncontrolled T2D patients on metformin and sulfonylurea on highest tolerable doses was associated with marked reductions in FPG and PPG and improved overall glycaemic control with high proportions of patients achieving optimal HbA1c targets safely. The postprandial effect was more pronounced. Lixisenatide treatment also resulted in notable reductions in body weight in a population that had a notably high BMI $\left(\geq 35 \mathrm{~kg} / \mathrm{m}^{2}\right)$ before treatment.

In conclusion, lixisenatide as add-on therapy to OADs in real-life settings has led to significant improvements in glycaemic control and has been associated with low incidence of hypoglycaemia and beneficial weight loss. Lixisenatide was well tolerated with few patients having adverse events or discontinuing therapy. Overall, these findings are consistent with lixisenatide's established safety and efficacy profile.

\section{ACKNOWLEDGEMENTS}

The authors thank the participants of the study and all investigators who contributed to patient recruitment and data collection.
Funding. Sponsorship for this study and article processing charges were funded by Sanofi Bulgaria. All authors had full access to all of the data in this study and take complete responsibility for the integrity of the data and accuracy of the data analysis.

Authorship. All named authors meet the International Committee of Medical Journal Editors (ICMJE) criteria for authorship for this manuscript, take responsibility for the integrity of the work as a whole, and have given final approval for the version to be published.

Disclosures. Natalyia Temelkova declares associations (member of advisory board, lecturer, clinical trial investigator) with the following companies: Amgen, AstraZeneca, Berlin Chemie, Boehringer Ingelheim, Eli Lilly, Merck Sharpe \& Dohme, Merck Serono, Novartis, Novo Nordisk, Sanofi and Servier. Stefka Vladeva declares associations (lecturer, clinical trial investigator) with Novo Nordisk, Eli Lilly, Sanofi, AstraZeneca, Boehringer Ingelheim, Servier, Merck Serono, Berlin Chemie and Mundipharma. Aleksi Delchev declares associations (lecturer, clinical trial investigator) with Novo Nordisk, Sanofi, Eli Lilly, Boehringer Ingelheim, AstraZeneca, Servier, Woerwag Pharma, Merck Serono and Milan. Kalina Ivanova declares associations (lecturer, clinical trial investigator) with AstraZeneca, Boehringer Ingelheim, Sanofi, Novo Nordisk and Eli Lilly. Yoanna Gerasimova-Zheleva declares associations (lecturer, clinical trial investigator) with Novo Nordisk, Sanofi, Boehringer Ingelheim and AstraZeneca. Tsvetodara Kuneva declares associations (lecturer, clinical trial investigator) with Novo Nordisk, Eli Lilly, Boehringer Ingelheim, Astra Zeneca and Servier. Veselina Pehlivanova is an employee of Sanofi. Plamen Popivanov declares associations declares associations (member of advisory board, lecturer, clinical trial investigator) with the following companies: AstraZeneca, Berlin Chemie, Boehringer Ingelheim, Amgen, Novo Nordisk, Sanofi, Eli Lilly, Merck Sharpe \& Dohme, Merck Serono, Novartis and Servier. 
Compliance with Ethics Guidelines. The study was conducted in accordance with the principles laid down by the 18th World Medical Assembly (Helsinki, 1964) including all subsequent amendments. It was approved by the regulatory authorities and the independent ethics committee in Bulgaria. Informed consent was obtained from all individual participants included in the study.

Data Availability. Qualified researchers may request access to patient-level data and related study documents, including the clinical study report, study protocol with any amendments, blank case report form, statistical analysis plan, and dataset specifications. Patientlevel data will be anonymized and study documents will be redacted to protect the privacy of trial participants. Further details on Sanofi's data sharing criteria, eligible studies, and process for requesting access can be found at https://www.clinicalstudydatarequest.com.

Open Access. This article is distributed under the terms of the Creative Commons Attribution-NonCommercial 4.0 International License (http://creativecommons.org/licenses/ by-nc/4.0/), which permits any noncommercial use, distribution, and reproduction in any medium, provided you give appropriate credit to the original author(s) and the source, provide a link to the Creative Commons license, and indicate if changes were made.

\section{REFERENCES}

1. Wild S, Roglic G, Green A, et al. Global prevalence of diabetes: estimates for the year 2000 and projections for 2030. Diabetes Care. 2004;27:1047-53.

2. Inzucchi SE, Bergenstal RM, Buse JB, et al. Management of hyperglycemia in type 2 diabetes, 2015: a patient-centered approach: update to a position statement of the American Diabetes Association and the European Association for the Study of Diabetes. Diabetes Care. 2015;38(1):140-9.

3. Davies MJ, D'Alessio DA, Fradkin J, et al. Management of hyperglycaemia in type 2 diabetes, 2018. A consensus report by the American Diabetes Association (ADA) and the European Association for the
Study of Diabetes (EASD). Diabetes Care. 2018;41(12): 2669-2701. https://doi.org/10.2337/ dci18-0033.

4. Thrasher J (2017) Pharmacologic management of type 2 diabetes mellitus: available therapies. Am J Med. 130:S4-S17. https://doi.rg/10.1016/j.amjmed. 2017.04.004.

5. Petersen $\mathrm{AB}$, Christensen M. Clinical potential of lixisenatide once daily treatment for type 2 diabetes mellitus. Diabetes Metab Syndrome Obes. 2013;6:217-31.

6. Werner U. Effects of the GLP-1 receptor agonist lixisenatide on postprandial glucose and gastric emptying-preclinical evidence. J Diabetes Complicat. 2014;28:110-4.

7. Htike Z, Zaccardi F, Papamargaritis D, et al. Efficacy and safety of glucagon-like peptide-1 receptor agonists in type 2 diabetes: a systematic review and mixed-treatment comparison analysis. Diabetes Obes Metab. 2017;19:524-36.

8. Sherman RE, et al. Real-world evidence-what is it and what can it tell us? $\mathrm{N}$ Engl J Med. 2016;375(23):2293-7.

9. Rosenstock J, Hanefeld M, Shamanna P, et al. Beneficial effects of once-daily lixisenatide on overall and postprandial glycemic levels without significant excess of hypoglycemia in type 2 diabetes inadequately controlled on a sulfonylurea with or without metformin (GetGoal-S). J Diabetes Compl. 2014;28:386-92.

10. Ahrén B, Dimas AL, Miossec P, et al. Efficacy and safety of lixisenatide once-daily morning or evening injections in type 2 diabetes inadequately controlled on metformin (GetGoal-M). Diabetes Care. 2013;36:2543-50.

11. Bolli G, Munteanu M, Dotsenko S, et al. Efficacy and safety of lixisenatide once-daily versus placebo in patients with type 2 diabetes mellitus insufficiently controlled on metformin (GetGoal-F1). Diabet Med. 2014;31:176-84.

12. Pinget $M$, Goldenberg R, Niemoeller E, et al. Efficacy and safety of lixisenatide once daily versus placebo in type 2 diabetes insufficiently controlled on pioglitazone (GetGoal-P). Diabetes Obes Metab. 2013;15:1000-7.

13. Carls GS, et al. Understanding the gap between efficacy in randomized controlled trials and effectiveness in real-world use of GLP-1 RA and DPP-4 therapies in patients with type 2 diabetes. Diabetes Care. 2017;40(11):1469-78. 
14. Feher M, Vega-Hernandez G, Mocevic E, et al. Effectiveness of liraglutide and lixisenatide in the treatment of type 2 diabetes: real-world evidence from the health improvement network (THIN) database in the United Kingdom. Diabetes Therapy. 2017;8:417-31.
15. Buysman EK, Liu F, Hammer M, Langer J. Impact of medication adherence and persistence on clinical and economic outcomes in patients with type 2 diabetes treated with liraglutide: a retrospective cohort study. Adv Ther. 2015;32:341-55. 